\title{
Performance Analysis of FSO under Turbulent Channel Using OSTBC
}

\author{
Lwaa Faisal Abdulameer \\ Information and Communication Eng. Dep. \\ University of Baghdad, Baghdad, IRAQ \\ lwaakhamass@gmail.com
}

Received: 18-Oct-2017 http://doi.org/10.29194/NJES.21030344

\author{
Hala Fadhil \\ Information and Communication Eng. Dep. \\ Al-Nahrain University, Baghdad, IRAQ \\ enghala302@yahoo.com \\ Revised: 05-Feb.-2018 Accepted: 30-April-2018
}

\begin{abstract}
Free Space Optics (FSO) plays a vital role in modern wireless communications due to its advantages over fiber optics and RF techniques where a transmission of huge bandwidth and access to remote places become possible.

The specific aim of this research is to analyze the Bit-Error Rate (BER) for FSO communication system when the signal is sent the over medium of turbulence channel, where the fading channel is described by the Gamma-Gamma model. The signal quality is improved by using Optical Space-Time Block- Code (OSTBC) and then the BER will be reduced. Optical $2 \times 2$ Alamouti scheme required $14 \mathrm{~dB}$ bit energy to noise ratio $\left(\mathrm{E}_{\mathrm{b}} / \mathrm{N}_{0}\right)$ at $10^{-5}$ bit error rate (BER) which gives $3.5 \mathrm{~dB}$ gain as compared to no diversity scheme.

The results show that using Multiple-InputMultiple-Output (MIMO) technique represented by Alamouti scheme gives the improved BER performance as compared with no diversity (Single-Input-Single-Output (SISO)) technique.
\end{abstract}

Keywords: FSO, OSTBC, Gamma-Gamma, MIMO

\section{Introduction}

FSO was initially created by US military and NASA being utilized recently in different systems to provide fast communication links. These systems offer automatic power-level control, and eliminate short-distance optical saturation. FSO is a wireless technology that transfer information through a free space medium where a modulated light beam is used as a carrier for data rather than RF signals [1]. In FSO system, different modulation schemes are used to modulate information signal at source such as ON-OFF Keying (OOK), Pulse Time Modulation Pulse Time Modulation (PTM), Polarization Shift Keying (PoSK). Each FSO system uses optical transmitter for transmit information towards destination and in receiving side high sensitive photodetector is used. But due to free space transmission, the attenuation caused by the atmosphere is major challenge faced by optical wireless communications which affect the performance of the link. The other factors which can affect the FSO are aerosols, beam scintillation, spreading and wandering signals absorption, and smoke [2].

To mitigate theses effects and improve the performance of overall system, MIMO is a suitable optical antennatechnology for wireless communications in which multiple antennas are used at transmitter as well as at receiver. The telescopes at each transmitter and receiver are combined to reduce BER and improve the data rate [3].

According to the optimization of the Alamouti Space Time Block Code (STBC) presented by Simon in [4], by using the complement signals, a special approach is considered in Space-TimeCode (STC) to match the optical requirements where the transmitted signal must be positive anyway [5-7]. To the best of the authors' knowledge, Diversity technique in FSO communications is considered widely in [8].

In this paper, On-Off Keying (OOK) is used to adapt STBC to optical signal where IM/DD is used as a modulation technique [9].

Our performance metric is the average BER where statistical characteristics for medium turbulence conditions modeled by (gammagamma distribution) and derived for two optical transmitting antennas and two optical receiving antennas taking in consideration the characteristics of optical signals.

In the following pages, section 2 presents the analysis of MIMO-FSO communication system. In section 3, the BER is analyzed and derived for the current system. In section 4 numerical results for $2 \times 2,2 \times 1$ Alamouti and No diversity schemes as well as the BER performance for selected modulation scheme (OOK) are computed where the numerical results is verified by Monte Carlo simulation.

\section{Analysis of MIMO FSO Communication System}

Let us now describes the block diagram in figure (1). OOK is a type of IM scheme where the bits ' 0 ' and ' 1 ' are represented by the presence and absence light. OOK is used in this work for simplicity although it is more sensitive to atmospheric effects. 

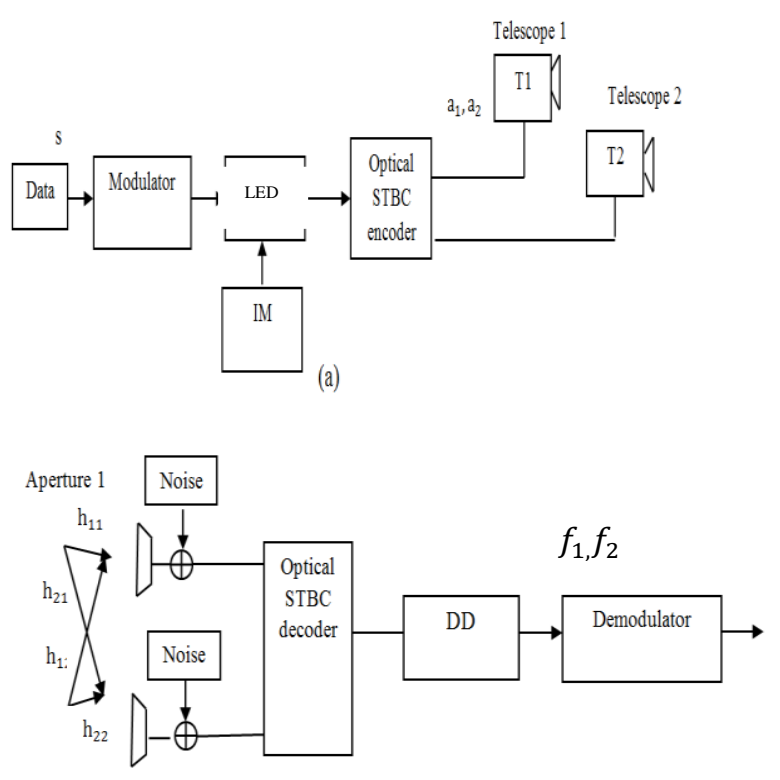

Aperture 2

(b)

Figure 1: FSO communication system, a) transmitter with two antennas, b) receiver with two antennas. IM: Intensity Modulation and DD: Direct Detection.

In above block diagram, consider a binary Intensity Modulation (IM) where $x_{0}$ represents symbol ' 0 ' and $x_{1}$ represents symbol ' 1 '.

Consider the information symbols $\left(s_{l} \in\{0,1\}\right)$ with symbol interval $T_{S}$. The data passed through IM block in figure (1) and sent to space-time block are from the set $\{0, \mathrm{I}\}$, where $I$ represents the intensity of the optical source at emitter for symbol 1.

$\begin{array}{ll}\mathrm{s}_{0}(\mathrm{t})=0, & 0 \leq \mathrm{t} \leq T_{S} \\ \mathrm{~s}_{1}(\mathrm{t})=\mathrm{I}, & 0 \leq \mathrm{t} \leq T_{S}\end{array}$

The transmitted signal at the output of the emitter is:

$\mathrm{u}(\mathrm{t})=2 P_{T}(t) \sum_{\mathrm{l}=0}^{\infty} \mathrm{s}_{\mathrm{l}}$

Where $P_{T}$ is the radiated power transmitted by the LED. Then, the received signal is

$$
\mathrm{r}(\mathrm{t})=h \mathrm{Ru}(\mathrm{t})+\mathrm{z}(\mathrm{t})
$$

In equation (4) $h$ represents the channel gain, $R$ is the responsivity of the photodetector and $z(t)$ is the AWGN with zero mean and PSD equal to $Z=2 q I_{B}$ where $Z$ represents the noise power, $q$ and $I_{B}$ are the electron charge and the photocurrent generated by background light respectively.

The received signal is demodulated by the replica of the local sequence, and then integrated over $T_{s}$. Finally data are estimated by computing the sign of the decision variable at the output of correlator,

$$
D s_{1}=\operatorname{sign}\left(s_{1}+w_{l}\right)
$$

where the $\operatorname{sign}($.$) is the sign operator and \mathrm{w}_{\mathrm{l}}$ is a noise components after correlator. Let,

$s_{i}(t)=-s_{j}(t)+I, \quad i \neq j, \quad i, j \in\{0,1\} \ldots(6)$ $\bar{f}_{l}$ is the complement of signal $f_{i}$ that represents the complement binary state of the signal $f_{i}$ (i.e., if $f_{i}=s_{0}$ then $\bar{f}_{l}=s_{1}$ and if $f=s_{1}$ then $\left.\bar{f}_{l}=s_{0}\right)$.

Stratify the above criteria to equation (6) leads to the following relationship,

$\bar{f}(t)=-f(t)+I, \quad f(t) \in\left\{s_{0}, s_{1}\right\}$

We can note that $f(t)=0, I$ the relationship in equation (7) ensure that $f(t)$ is non-negative. The primary property of a space-time code $F\left(f_{1}, \ldots \ldots \ldots, f_{a}\right)=F(x) \quad$ with $\quad f=$ $\left(f_{1}, \ldots \ldots \ldots f_{a}\right)^{T}$ is

$F^{T}(f) F(f)=I_{A}\|f\|^{2}$

Where, $(*)^{T}$ denotes transpose operation, $I_{A}$ is the $F \times F$ unit matrix, $F$ is the number of transmitter antennas and $\|f\|^{2}=\left(f_{1}^{2}+f_{2}^{2}+\cdots+f_{a}^{2}\right)$.

However, a coding scheme satisfying (8) cannot be implemented in IM/DD system because transmitted IM signal must be non-negative at all times.

The coding scheme in the above equations shows that the transmitter outputs must be negated to match the orthogonality.

To solve the above problem, we use the matrix in equation (9)

$(|F|(f, \bar{f}))_{i, j}$

$=\left\{\begin{array}{c}(F(f))_{i, j} \text { if }\left(F\left(f_{1}=1, \ldots \ldots \ldots f_{a}=1\right)\right)_{i, j} \geq 0 \\ -(F(f))_{i, j} \text { otherwise } \ldots \ldots(9)\end{array}\right.$

This equation ensures that the emitted symbols are always positive for an IM/DD system. The estimated signal at receiver is

$\tilde{f}=F^{T}(h) J_{P} \times\left(J_{P} F(h) x+z\right)$

Using the relation $\mathrm{J}_{\mathrm{P}} \times \mathrm{J}_{\mathrm{P}}=\mathrm{I}_{\mathrm{P}}$, we obtain:

$\tilde{f}=\|\mathrm{h}\|^{2} \mathrm{x}+\mathrm{F}^{\mathrm{T}}(\mathrm{h}) \mathrm{J}_{\mathrm{P}} \mathrm{m}$

Where $P$ represents the transmission time slots, $\mathrm{J}_{\mathrm{P}}$ is $\mathrm{P} \times \mathrm{P}$ matrix with $\mathrm{J}=$ $\operatorname{diag}(1,-1,-1,-1, \ldots,-1), \quad \bar{x}=$ $\left(\overline{x_{1}} \ldots \ldots \ldots . \overline{x_{a}}\right)^{T}, \quad m=\left(m \ldots \ldots \ldots m_{P}\right)^{T}$ are Gaussian noise, which may include contribution from thermal and/or shot noise and $h=$ $\left(h_{1} \ldots \ldots \ldots h_{a}\right)^{T}$ is the quasi-static channel response for transmitters $i=1,2, \ldots \ldots \ldots a$ respectively $[10,11]$.

Then, the optical Alamouti matrix becomes

$$
X(x, \bar{x})=\left(\begin{array}{ll}
f_{1} & f_{2} \\
\overline{f_{2}} & f_{1}
\end{array}\right)
$$

This matrix is considered in optical communication to overcome the negative values 
already considered in the matrix of traditional STBC where the power must be non-negative.

For $2 \times 1$ optical Alamouti, the estimated signal at receiver

$\left(\begin{array}{l}\widetilde{f}_{1} \\ \widetilde{f}_{2}\end{array}\right)=\left(h_{1}^{2}+h_{2}^{2}\right)\left(\begin{array}{l}f_{1} \\ f_{2}\end{array}\right)+\left(\begin{array}{l}h_{1} z_{1}+h_{2} z_{2} \\ h_{2} z_{1}-h_{1} z_{2}\end{array}\right)$

Where $h=\left(h_{1}, h_{2}\right)^{T}$ is the channel gain for two lasers (antennas).

For 2 transmit antennas and 2 receive antennas and according to equation (12), the estimated signal at receiver

$$
\begin{aligned}
\left(\begin{array}{l}
\widetilde{f}_{1} \\
\widetilde{f}_{2}
\end{array}\right)=\left(h_{11}^{2}+h_{21}^{2}+h_{12}^{2}\right. & \\
& \left.+h_{22}^{2}\right)\left(\begin{array}{l}
f_{1} \\
f_{2}
\end{array}\right)\left(\begin{array}{l}
h_{11} z_{1}+h_{21} z_{2} \\
h_{22} z_{1}-h_{12} z_{2}
\end{array}\right)
\end{aligned}
$$

Table 1: The design of the emitted signal of $2 \times 2$ Alamouti.

\begin{tabular}{|l|c|c|}
\hline Time & $f_{1}(t)$ from Telescope & $f_{2}(t)$ from Telescope \\
\hline 1st time slot & $f_{1} u_{i}(\mathrm{t})$ & $f_{2} u_{i}(\mathrm{t})$ \\
\hline 2nd time slot & $\bar{f}_{2} u_{i+\text { time slot }}(\mathrm{t})$ & $f_{1} u_{i+\text { time slot }}(\mathrm{t})$ \\
\hline
\end{tabular}

Table 2: The design of received signal on aperture 1

\begin{tabular}{|l|c|}
\hline Time & Received signal on aperture 1 \\
\hline 1 st time slot & $2 P_{T} R\left[h_{11} u_{i}(\mathrm{t}) f_{1}+h_{21} u_{i}(\mathrm{t}) f_{2}\right]+Z_{11}$ \\
\hline 2nd time slot & $2 P_{T} R\left[h_{11} u_{i+\text { time slot }}(\mathrm{t}) \overline{f_{2}}\right.$ \\
& $\left.+h_{21} u_{i+\text { time slot }}(\mathrm{t}) f_{1}\right]+Z_{21}$ \\
\hline
\end{tabular}

Table 3: The design of received signal on aperture 2

\begin{tabular}{|l|c|}
\hline Time & Received signal on aperture 2 \\
\hline 1st time slot & $2 P_{T} R\left[h_{12} u_{i}(\mathrm{t}) f_{1}+h_{22} u_{i}(\mathrm{t}) f_{2}\right]+Z_{12}$ \\
\hline 2nd time slot & $2 P_{T} R\left[h_{12} u_{i+\text { ime slot }}(\mathrm{t}) \bar{f}_{2}\right.$ \\
& $\left.+h_{22} u_{i+\text { ime slot }}(\mathrm{t}) f_{1}\right]$ \\
& $+Z_{22}$ \\
\hline
\end{tabular}

Table 4: The baseband of the received signals on the aperture 1

\begin{tabular}{|l|l|}
\hline Time & $\begin{array}{l}\text { The baseband of the received signals on the } \\
\text { aperture } 1\end{array}$ \\
\hline 1st time slot & $V_{11}=2 P_{T}^{2} R \quad\left(h_{11} f_{1}+h_{21} f_{2}\right)+Z_{11}$ \\
\hline 2nd time slot & $V_{21}=2 P_{T}^{2} R \quad\left(h_{11} \bar{f}_{2}+h_{21} f_{1}\right)+Z_{21}$ \\
\hline
\end{tabular}

Where $Z_{11}$ and $Z_{21}$ are the components of the noise.

Table 5: The baseband of the received signals on the aperture 2

\begin{tabular}{|l|c|}
\hline Time & $\begin{array}{l}\text { The baseband of the received signals on the } \\
\text { aperture } 2\end{array}$ \\
\hline 1st time slot & $V_{12}=2 P_{T}^{2} R \quad\left(h_{12} f_{1}+h_{22} f_{2}\right)+Z_{12}$ \\
\hline 2nd time slot & $V_{22}=2 P_{T}^{2} R \quad\left(h_{12} \bar{f}_{2}+h_{22} f_{1}\right)+Z_{22}$ \\
\hline
\end{tabular}

where $Z_{12}=Z_{11}$ and $Z_{22}=Z_{21}$.

The channel model is written as,

$\mathrm{V}=2 P_{T}^{2} R \mathrm{HF}+\mathrm{Z}$

Then, the received signal for $2 \times 2$ Alamouti

$$
\begin{aligned}
& \left(\begin{array}{l}
V_{11} \\
V_{12} \\
V_{21}^{*} \\
V_{22}^{*}
\end{array}\right)=2 P_{T}^{2} R\left(\begin{array}{ll}
h_{11} & h_{21} \\
h_{12} & h_{22} \\
h_{21}^{*} & \tilde{h}_{11}^{*} \\
h_{22}^{\wedge} & \tilde{h}_{12}^{*}
\end{array}\right)\left(\begin{array}{l}
f_{1} \\
f_{2}
\end{array}\right)+ \\
& \left(\begin{array}{l}
Z_{11} \\
Z_{12} \\
Z_{21}^{*} \\
Z_{22}^{*}
\end{array}\right)
\end{aligned}
$$

The emitted bits are reconstructed by multiplying the received signal $\mathrm{V}$ by the conjugate transpose of the matrix $H$ :

$$
\left(\begin{array}{l}
D_{s 1} \\
D_{s 2}
\end{array}\right)=H^{*} V
$$

The estimated bits can be computed from the sign of the decision variables,

$$
\begin{aligned}
& D_{s 1}=2 P_{T}^{2} R \quad f_{1}\left(h_{11}^{2}+h_{21}^{2}+h_{12}^{2}+h_{22}^{2}\right)+ \\
& \left(h_{11}+h_{12}+h_{21}^{*}+h_{22}^{*}\right) Z \\
& D_{s 2}=2 P_{T}^{2} R f_{2}\left(h_{11}^{2}+h_{21}^{2}+h_{12}^{2}+h_{22}^{2}\right)+ \\
& \left(h_{21}+h_{22}+\tilde{h}_{11}^{*}+\tilde{h}_{12}^{*}\right) Z
\end{aligned}
$$

Then, the emitted bits are

$f_{1}=\operatorname{sign} D_{s 1} ; f_{2}=\operatorname{sign} D_{s 2}$

In the turbulence channel model, the radiated power is multiplied by the irradiance of channel as a noise model which is substituted mathematically by two random variables independent on each other that is, $\mathrm{p}(I)=\mathrm{p}(I x) \mathrm{p}(I y), I x$ represents the large scale and $I y$ represents the small scale. This leads to the so-called gamma-gamma model, whose PDF is given by,

$$
f_{I}\left(I_{m n}\right)=\frac{2(\alpha \beta)^{\frac{\alpha+\beta}{2}}}{\Gamma(\alpha) \Gamma(\beta)} I_{m n}^{\frac{\alpha+\beta}{2-1}} K_{\alpha-\beta}\left(2 \sqrt{\alpha \beta I_{m n}}\right)
$$

Where:

$\alpha>0$ and $\beta>0$. So, the scintillation index $\triangleq$ $\frac{1}{\alpha}+\frac{1}{\beta}+\frac{1}{\alpha \beta}$ and $\Gamma($.$) is the gamma function.$

Where:

$\alpha=\left[\exp \left(\frac{0.49 x^{2}}{\left(1+0.18 d^{2}+0.56 x^{12 / 5}\right)^{7 / 6}}\right)-1\right]^{-1}$
$\beta=\left[\exp \left(\frac{0.51 x^{2}\left(1+0.69 x^{12 / 5}\right)^{-5 / 6}}{\left(1+0.9 d^{2}+0.62 d^{2} x^{12 / 5}\right)^{5 / 6}}\right)-1\right]^{-1}$

Where:

$\mathrm{x}^{2}=0.5 * C_{n}^{2} * K^{\frac{7}{6} *} * L^{11 / 6}, d=\left(\frac{K D^{2}}{4 L}\right)^{1 / 2}$, and $k=$ $\frac{2 \pi}{\lambda}$ is the wave number and $\lambda, D, C_{n}^{2}$, and $L$ are the wavelength, diameter of the receiver collecting lens aperture, refraction index structure parameter and $\mathrm{L}$ is the distance between emitter and receiver respectively. The parameters used in this work are [8],

$\lambda=1550 \mathrm{~nm}$ where the loss is minimum. $\mathrm{D}=0.02$

m. $\mathrm{C}=10^{-15}$ for medium turbulence. $\mathrm{L}=1 \mathrm{Km}$. 
NJES Vol.21, No.3, 2018

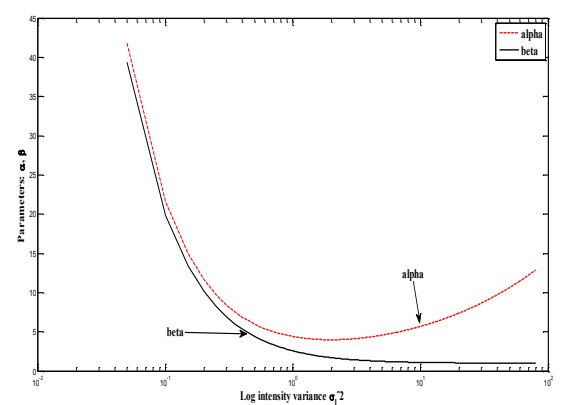

Figure 2: Values of $\alpha$ and $\beta$ from weak turbulence to strong turbulence.

\section{BER Performance Analysis}

The decision variables of both $D_{s 1}$ and $D_{s 2}$ are the same, so the statistical properties are also the same. Then we can compute the mean and variance of the random variables for one of them.

The elements of noise components are zero mean and independent. So equation (18) can be minimized to

$E\left[D_{s 1}^{(l)}\right]=2 P_{T}^{2} R f_{1}^{(l)}\left(h_{11}^{2}+h_{21}^{2}+h_{12}^{2}+h_{22}^{2}\right)$

The variance var[.] of the $D_{s 1}$ can be computed as

$v\left[D_{s 1}^{(l)}\right]=E\left[\left(D_{s 1}\right)^{2}\right]-E\left[D_{s 1}\right]^{2}=E\left[f_{1} P_{T}^{2}\left(h_{11}^{2}+\right.\right.$ $\left.\left.\left.h_{21}^{2}+h_{12}^{2}+h_{22}^{2}\right)\right\}^{2}\right]-\left[f_{1} P_{T}^{2}\left(h_{11}^{2}+h_{21}^{2}+h_{12}^{2}+\right.\right.$ $\left.\left.h_{22}^{2}\right)\right]^{2}+E\left[\left\{\left(h_{11}+h_{12}+h_{21}^{*}+h_{22}^{*}\right) Z\right\}^{2}\right.$

Since the samples of gaussian noise are independent and uncorrelated, the variance of $D_{s 1}$ for a given $l t h$ bit is

$\sigma_{D_{S 1}}^{2}=\operatorname{var}\left[h_{11} Z_{11}\right]+\operatorname{var}\left[h_{12} Z_{12}\right]+$

$\operatorname{var}\left[h_{21}^{*} Z_{21}^{*}\right]+\operatorname{var}\left[h_{22}^{*} Z_{22}^{*}\right]$

Then,

$\sigma_{1}^{2}=\operatorname{var}\left[h_{11} Z_{11}\right]=\operatorname{var}\left[h_{11} \sum_{k=1}^{W} z_{l}^{k}\right]$,

Where $W$ is an integer and $\sum z_{l}^{k}$ is a sumation of a large number of the noise components. Then,

$$
\sigma_{1}^{2}=h_{11} \frac{Z}{2}
$$

by analogy, the variances $\sigma_{2}^{2}, \sigma_{3}^{2}$ and $\sigma_{4}^{2}$ are given as follows:

$\sigma_{2}^{2}=h_{12} \frac{Z}{2}, \sigma_{3}^{2}=h_{21} \frac{Z}{2}, \sigma_{4}^{2}=h_{22} \frac{Z}{2}$,

Then, the total variance can be computed as,

$$
\begin{aligned}
& v\left[D_{s 1}^{(l)}\right]=\left(h_{11}^{2}+h_{21}^{2}+h_{12}^{2}+h_{22}^{2}\right) \frac{Z}{2} \\
& P_{e}\left(P_{T}^{2}\right)=\frac{1}{2} \operatorname{erfc}\left[\frac{E\left[D_{s 1}^{(l)}\right]}{\left.\sqrt{2 v\left[D_{s 1}^{(l)}\right.}\right]}\right]
\end{aligned}
$$

As a result, this error probability for $2 \times 2$ Alamouti is given by

$$
P_{e}\left(P_{T}^{2}\right)=\frac{1}{2} \operatorname{erfc} \sqrt{\left[\frac{2 P_{T}^{2} R\left(h_{11}^{2}+h_{21}^{2}+h_{12}^{2}+h_{22}^{2}\right)}{Z}\right]}
$$

Abdulameer \& Fadhil, pp.344-349

Where $h_{i j}$ in equation (28) is not constant and represents the channel gain of Gamma-Gamma fading channel. So if $h_{i j}$ is constant and equal to 1 , it represents AWGN assumption.

\section{Results and Discussions}

One interesting computing the BER of OOKNRZ, where the radiated power $P_{T}$ is equal to 10 $\mathrm{mW}$ and photodetector responsivity is equal to 1 . It is observed in figure (3) that the blue graph represent BER curve for simulated OOK-NRZ which is a large extent align with the red curve which represent theoretical BER.

The graph in figure (4) shows a BER performance comparison of different schemes (SISO (No Diversity) and Alamouti ( $2 \times 1$ and $2 \times 2)$ ) schemes for FSO communication system under medium turbulent channel $\left(\mathrm{C}=10^{-15}\right)$ represented by Gamma-Gamma fading channel.

Figure (4) reveals that a $2 \times 2$ Alamouti scheme gives the superior BER performance when compared with $2 \times 1$ scheme and No Diversity communication schemes. $2 \times 2$ Alamouti scheme gives $1.5 \mathrm{~dB}$ gain as compared to $2 \times 1$ and $3.5 \mathrm{~dB}$ gain at $10^{-5} \mathrm{BER}$ as compared to No diversity schemes under the same conditions.

These results reveals that the BER performance of optical Alamouti communication systems $(2 \times 1$ and $2 \times 2)$ is the best as compared to the performance offered by no diversity. Hence, a combined system gives acceptable BER under Gamma-Gamma fading.

Traditional and important problem presented in figure (5) where the power requirements for any communication scheme increases when we need more bandwidth. It is clear that the OOK perform better than PPM modulation schemes in this context for proposed system where it is required less power to provide more bandwidth.

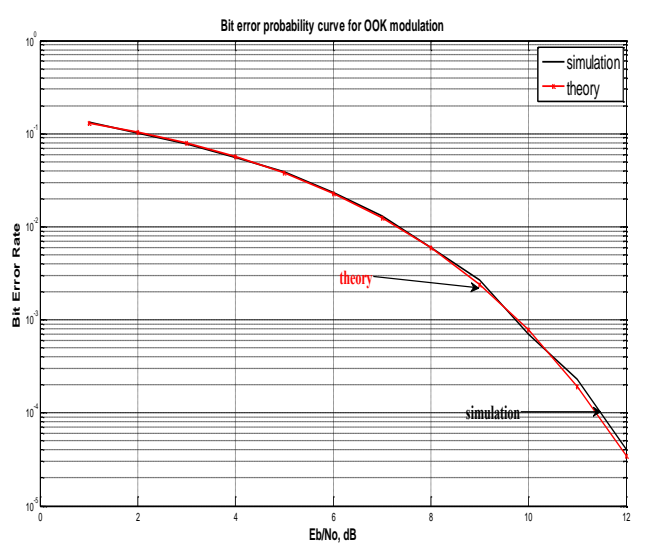

Figure 3: BER vs. Eb/NO of OOK-NRZ for FSO communication system 


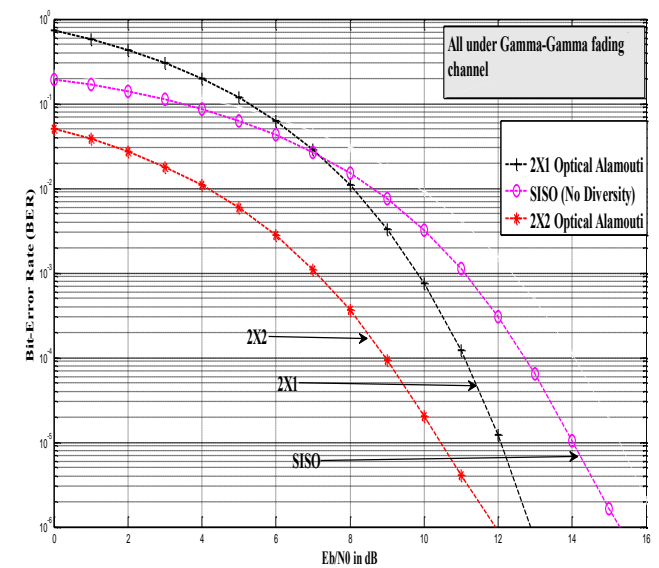

Figure 4: BER performances for different communication schemes using $2 \times 2, \quad 2 \times 1$ Alamouti and No diversity under Gamma-Gamma fading channel

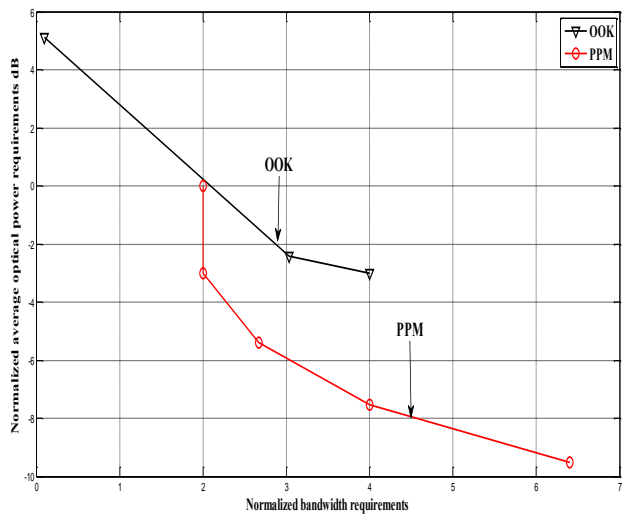

Figure 5: power requirement Vs. bandwidth

\section{Conclusions}

In this work, we designed an FSO communication system combined with Alamouti scheme using OOK modulation format to mitigate the BER due to the atmospheric turbulence. We have been concluded that $2 \times 2$ Alamouti gives a good BER performance as compared to $2 \times 1$ Alamouti and both gives the best BER performance as compared to no diversity communication system under Gamma-Gamma fading channel. Mathematical analysis for the FSO MIMO communication system has been derived and achieved using Matlab.

\section{References}

[1] M. J. Lok1, I. E. Lee1, Z. Ghassemlooy2, and G. C. Chung1, " Investigation on the Error Performance of Modulation Formats in Freespace Optical Communication Links with
Turbulences", 10th International Symposium on Communication Systems, Networks and Digital Signal Processing (CSNDSP), 2016.

[2] G. Kaur1, H. Singh2, Amandeep Singh Sappal, " Free Space Optical Using Different Modulation Techniques - $\quad$ A Review",International Journal of Engineering Trends and Technology (IJETT) - Volume-43 Number-2, January 2017.

[3] T. Nara, N. Joshi, S. Ahuja, "Performance of Free-Space Optical MIMO system in SDistributed Turbulence". International Conference on Computing, Communication and Automation (ICCCA) 2016.

[4] M. Simon and V. Vilnrotter, "Alamouti-type space-time coding for freespace optical communication with direct detection," IEEE Trans. Wireless Commun., vol. 4, no. 1, pp. 35392005 .

[5] A. Garc'1a-Zambrana and A. Puerta-Notario, "Novel approach for increasing the peak-toaverage optical power ratio in rate-adaptive optical wireless communications systems," IEE Proc. Optoelectron., vol. 150, no. 5, pp. 439-444, 2003.

[6] A. Garc'1a-Zambrana, " Error Rate Performance for STBC in Free-Space Optical Communications through Strong Atmospheric Turbulence", IEEE COMMUNICATIONS LETTERS, VOL. 11, NO. 5 MAY 2007.

[7] J. Abouei and K. N. Plataniotis, B., "Multiuser diversity scheduling in free-space optical communications, IEEE/OSA J.Lightw. Technol., vol. 30, no. 9, pp. 1351-1358 May 2012.

[8] L. Yang,X. Gao, Senior Member, IEEE, Mohamed-Slim Alouini, Fellow, IEEE, Performance Analysis of Free-Space Optical Communication Systems With Multiuser Diversity Over Atmospheric Turbulence Channels" ,Volume 6, Number 2 April 2014.

[9] M .Shinozuka., A. Uchida, Ogawa T. Yoshmori S. and Kannari F., "Chaotic on-off keying method in microchip lasers for secure communications 2001.

[10] Y. Hiroshi and O. Tomoaki,, " AlamoutiType Space-Time Coding for Free-Space Optical Communication with Direct Detection, 2003.

[11] L. Faisal., D. Jokhakar, U. Sripati and M. Kulkarni., "BER Performance Enhancement for Secure Wireless Optical Communication Systems Based on Chaotic MIMO Techniques". Nonlinear Dynamics, (SPRINGER), Vol. 75, p.p. 7-16 January 2014. 


\title{
تحليل اداء منظومة الاتصالات الضوئية اللاسلكية تحت تأثثر المعوقات الجوية

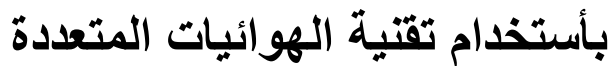

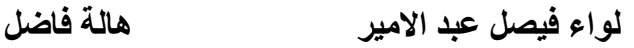

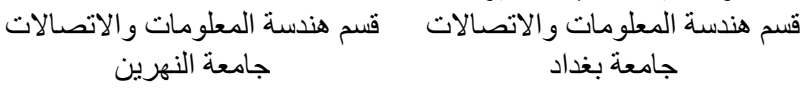

الخلاصة الغنة

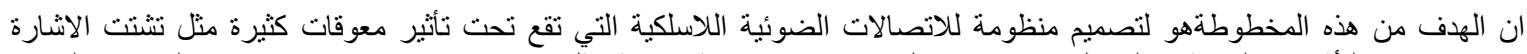

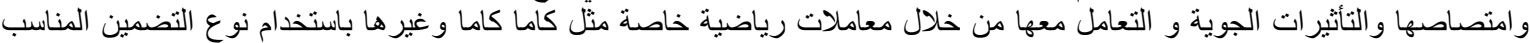

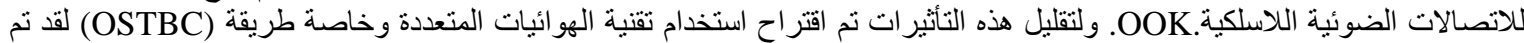

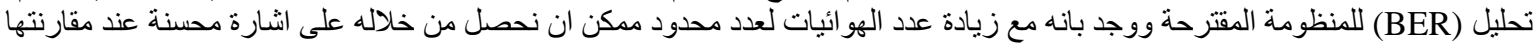

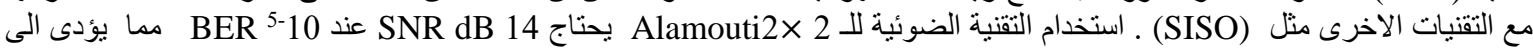
تحسين اداء المظومة بمقدار dB3.5 بالمقارنة مع المنظومة التقليدية. 